\title{
EL CORO DE ADOLESCENTES EN UN INSTITUTO DE EDUCACIÓN SECUNDARIA: UN ESTUDIO DE FONACIÓN
}

\author{
Alfonso Elorriaga \\ Universidad Complutense de Madrid \\ alfonsojesus.elorriaga@edu.ucm.es
}

\begin{abstract}
Este estudio presenta un método de clasificación de la voz en la adolescencia. Los datos cuantitativos revelan aspectos relacionados con la fonación. Los resultados apuntan a debatir los prejuicios imperantes sobre el canto en esta etapa de la vida. Se defiende la idea de que una formación vocal (con contenidos tanto prácticos como teóricos) continuada durante la muda de la voz mejoraría la actitud y la opinión que los adolescentes suelen mostrar hacia el canto coral en la educación secundaria.
\end{abstract}

This study shows a method for categorizing the singing voice during adolescence. Quantitative data focus on aspects concerned with phonation. Evidence leads us to discuss current prejudices about singing among adolescents. The author maintains that continuous vocal training (theoretical and practical) would help adolescents to have a better attitude toward and opinions about choral singing in secondary school.

\section{INTRODUCCIÓN}

En España existe un vacío importante acerca de la didáctica del canto en esta etapa específica de la vida, la adolescencia. Es muy difícil encontrar publicaciones especializadas en este tema, y en concreto el desconocimiento del proceso de la muda de la voz tanto en hombres como en mujeres está muy generalizado. Así las cosas, es fácil constatar la idea tan extendida de que en esta edad es mejor no cantar, o aceptar el hecho de que el resultado musical será siempre irremediablemente más pobre que durante la infancia. Teniendo en cuenta que el marco educativo legal de nuestro país ha incluido la expresión vocal en el currículo de la enseñanza musical en la etapa de educación secundaria obligatoria desde la aprobación de la Ley Orgánica General del Sistema Educativo (LOGSE) en 1990, llama poderosamente la escasa documentación publicada en nuestro país sobre la muda de la voz y su relación con la didáctica del canto coral en la pubertad. Actualmente, la reforma curricular de 2006 (MEC, 2006) propone en la asignatura de música en la etapa de la educación secundaria, un currículo dividido en bloques de contenidos, y en uno de ellos, el denominado "Interpretación”, incluye referencias a la voz como medio de expresión musical, pero sin llegar a mencionar en absoluto la muda de la voz a pesar de ser un factor condicionante de primer orden en el desarrollo y utilización de la voz en el aula de música de educación secundaria. Tampoco se encuentran referencias al respecto de este tema en el preámbulo de la asignatura. Hoy en día la educación secundaria es un tramo educativo donde la interpretación musical en el aula está más enfocada hacia la práctica instrumental: "Cuando la actividad musical tiene lugar, suele incluir tocar la flauta y los instrumentos Orff, y un mínimo de cantar, bailar o componer” (RUSINEK, 2008:13). Así las cosas, los alumnos cantan muy poco en el aula, e incluso rechazan esta actividad, como demuestran los estudios existentes hasta la fecha: "La percepción generalizada de que el canto va perdiendo espacio en el trabajo en el aula de música dentro del ámbito de la enseñanza general, a partir de ciertas edades, nos debe llevar a la reflexión sobre el papel que juega la práctica de cantar en los diferentes niveles del sistema educativo" (CÁMARA, 2004:9). Es obvio que si se pretende cambiar esta tendencia primero se debe investigar y sacar a la luz cuáles son los problemas específicos, fisiológicos y psíquicos, que aparecen en estas edades y ofrecer una serie de técnicas de intervención contrastadas que optimicen el desarrollo de la percepción y la expresión musical a través del canto durante el proceso de la muda de la voz.

De este modo se obtendrían unas bases sólidas que nos conducirían a la formulación de una serie de principios de trabajo que podrían tenerse en cuenta en la docencia musical durante la adolescencia. Establecidos los fines de la investigación, el principal objetivo de la misma era, por lo tanto, indagar tanto en el aspecto fisiológico y evolutivo de la voz adolescente como en el psicoló- 
gico. Con respecto a éste último, cabe decir que también se llevó a cabo otro estudio relacionado con la relación entre la identidad vocal de los alumnos varones y sus respectivas identidades de género (ELORRIAGA, 2010c). Sin embargo, y volviendo de nuevo a las preguntas que guiaron primigeniamente el aspecto fisiológico de la investigación, éstas quedaron planteadas del siguiente modo:

- ¿ ¿Qué grado de variabilidad hay en la fonación durante la muda de la voz masculina y femenina?

- ¿ ¿Qué factores externos pueden influir en las variables de categorización de la voz adolescente?

El conocimiento científico derivado de la investigación de campo en el terreno de la educación vocal escolar debería conducir a los profesores de educación secundaria a poseer una renovada y actualizada praxis docente que en cierta medida tienda puentes entre las metodologías ya existentes, la psicopedagogía musical y una nueva línea de investigación en relación con la voz en la adolescencia. "A lo largo de todos los momentos del desarrollo de la materia, conviene no olvidar que la vía de acceso al aprendizaje se realiza siempre desde la práctica. Los procedimientos y las actitudes serán, pues, la vía de acceso a su posterior conceptualización” (REY, 1992:25). "En un primer momento, lo único que se puede hacer es coger aspectos de los principios generales de las metodologías musicales del siglo XX y ponerlas en práctica desde la experiencia e intuición del docente encargado. De la misma manera hay que tener en cuenta, obviamente, las necesarias consideraciones de la psicología musical” (MuÑOZ, 2007:98). La presente investigación aspiraría a esclarecer los principios fundamentales que deben de ser tenidos en cuenta en el aprendizaje de la música durante la etapa de la muda de la voz. "La formulación y evaluación de teorías es fundamental en cualquier empresa científica. Las teorías son estructuras o marcos referenciales que nos permiten organizar hechos y verlos en perspectiva” (HARGREAVES, 1998:23).

\section{LA MUDA DE LA VOZ EN LA ADOLESCENCIA}

La voz humana ha sido estudiada tanto desde la perspectiva fisiológica como desde la psicológica. Ambos factores determinan su estudio y se complementan mutuamente. Desde los escritos del español Manuel Vicente GARCíA $(1847 ; 1894)$ en el siglo XIX (cantante y profesor de canto, inventor del laringoscopio) se conoce que las cuerdas vocales cuando se colocan en posición de fonación, se ajustan (es decir, entran en contacto) de dos modos (PHILlips, 1996): el ajuste vocal agudo, que produce la voz de cabeza, y el ajuste vocal grave, que da lugar a la voz de pecho. Ambos registros se mantienen a lo largo de todas las etapas de la vida en ambos sexos. Los primeros cambios corporales que se manifiestan en los niños comienzan con la pubertad, sobre los doce o trece años, los cuales se evidencian a través de la aparición de las características sexuales secundarias. Diversos factores (hormonales, genéticos, ambientales, nutricionales, etc.) inciden en el ritmo de crecimiento de cada adolescente, así como en la aspectos cualitativos de la maduración sexual, tanto del varón como de la mujer. Durante la adolescencia se produce el cambio o muda de la voz, en ambos sexos, lo cual va a propiciar un cambio cualitativo considerable en la voz hablada y cantada. "La adolescencia representa un periodo de cambios muy rápidos que afectan a las dimensiones biológicas, psicológicas y sociales de la persona” (CALVO y ROMÁN, 2007:38). Por otra parte, el proceso de la muda de la voz ha sido ampliamente estudiado y formulado tanto para hombres como para mujeres. En el caso de la muda de la voz masculina adolescente, los trabajos de COOKSEY (1977a, 1977b, 1977c, 1978) constituyen el principal punto de partida. Este investigador señala que "la muda de la voz es un proceso secuencial y predecible que generalmente ocurre durante un período de uno o dos años, entre los doce y los catorce normalmente” (COOKSEY, 1992:8). Para clasificar las voces con precisión, es posible diferenciar cinco fases de la muda de la voz masculina durante la pubertad de acuerdo con los siguientes factores:

1) SFF (speaking fundamental frequency): la frecuencia básica de la voz hablada (WILLIS y KENNY, 2007b) es un parámetro que mide la afinación habitual de la voz hablada, la que solemos 
usar con naturalidad cuando comenzamos a recitar, hablar o saludar (por ejemplo, diciendo "hola").

2) AFF (average fundamental frequency): parámetro similar al SFF, pero en forma de intervalo. Marca los límites agudo y grave del promedio de afinación habitual de la voz hablada (normalmente 1 semitono ascendente-descendente a partir del SFF), medidos con los índices acústicos del sistema de banda audible (Do4 como Do central del piano).

3) Ámbito o registro vocal: viene determinado por la nota más aguda del registro o ámbito (HTP: high terminal pitch) y la más grave posible (LTP: low terminal pitch) que un individuo puede emitir cantando sin forzar su laringe más allá del umbral de la molestia física o el dolor.

4) Tesitura: es el conjunto de notas que un individuo puede cantar con el mayor grado de calidad vocal posible de acuerdo a sus posibilidades. Se trata de un registro más corto que el ámbito, ya que se excluyen las notas terminales cercanas a los límites del LTP y HTP.

5) Cualidades vocales especialmente significativas en la adolescencia (COOKSEY, 1993):

a. resonancia de la voz: cantidad de armónicos presentes en la voz que entran en resonancia con las cavidades bucales (dando lugar a los formantes de la voz);

b. color de la voz: timbre de la voz (calidad de los armónicos);

c. aspereza de la voz: limpieza en la emisión, calidad del acoplamiento vocálico de las cuerdas;

d. ventilación de la voz: cantidad de aire fugado en la fonación;

e. constricción de la voz: grado de sobre-esfuerzo laríngeo y de la musculatura relacionada.

\section{La muda de la voz masculina}

Las etapas de la muda de la voz masculina (COOKSEY, 1992:10) son:

a) voz blanca, infantil (anterior a la muda de la voz).

b) mediavoz 1

c) mediavoz 2

d) mediavoz $2 \mathrm{a}$

e) nuevo barítono

f) barítono en desarrollo o barítono joven hacia la voz adulta (esta fase se añadió posteriormente).

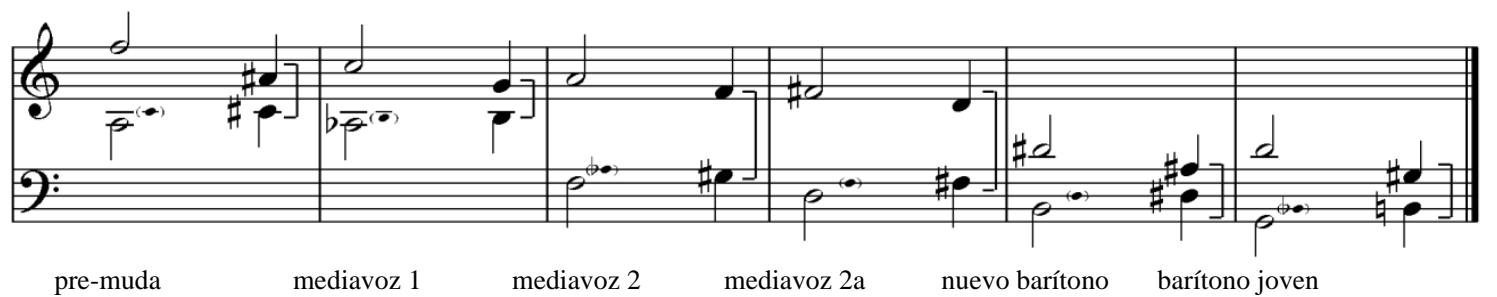

En cada compás, las blancas indican el ámbito vocal (HTP-LTP) y las negras entre corchetes indican la tesitura cantable. La nota entre paréntesis indica el SFF aproximado. Cada etapa posee sus propias características en cuanto a SFF, ámbito, tesitura y cualidades vocales que hacen de ella algo único y distinguible del resto. El ritmo y velocidad de la muda de la voz varía entre distintos individuos y ambos están directamente relacionados con el desarrollo de las características sexuales primarias y secundarias asociadas al periodo de la pubertad y otros factores hormonales propios de cada adolescente. Los primeros síntomas de la muda de la voz denotan un incremento de la ventilación de la voz y la aparición de ciertos rasgos de tensión vocal en el registro agudo de la voz infantil. Este hecho suele ocasionar una pérdida repentina del registro más agudo de la voz infantil como un rasgo característico que anuncia el comienzo inminente del proceso de la muda de la voz. Esta pérdida de notas agudas suele aparecer antes que comience a descender el registro grave de la voz, por ello, al principio de la muda, el ámbito vocal sufre un acortamiento en el agudo sin por ello presentar aún una expansión descendente en el grave. Una vez iniciado el proceso de la muda, ésta se 
hace notar porque el registro grave comienza a descender gradualmente. La nota más grave del ámbito vocal (LTP) muestra una progresión continua de terceras descendentes durante las cinco etapas de la voz adolescente masculina. Normalmente desciende uno o dos semitonos cada tres o cuatro semanas.

La voz del adolescente tiende a permanecer estable en torno al LTP de cada una de las etapas, mientras que el HTP de cada etapa se muestra más fluctuante, inestable e impredecible (BARLOW y HowARD, 2007). Finalmente, el HTP sigue igualmente la tendencia a ir progresando descendentemente, pero lo hace de un modo mucho más irregular que el LTP. Por lo tanto, el HTP es mucho más variable que el LTP. De lo dicho anteriormente se deduce que una vez iniciado el proceso de la muda de la voz, en cada una de las etapas la voz hablada cambia antes que la cantada, es decir, que normalmente tanto el SFF como el LTP descienden y posteriormente lo hace el HTP.

Todas las voces adolescentes sufren, en una amplia mayoría, de una pérdida generalizada de brillantez, plenitud, intensidad y redondez (WILLIS y KENNY, 2007a). Igualmente suelen presentar frecuentemente cierta pérdida de armónicos y por lo tanto se vuelven menos resonantes y más débiles. Son igualmente menos ágiles y más sensibles a un esfuerzo vocal continuado (CHAPMAN, 1989). El registro de falsete en la voz masculina, (en tanto que nueva voz de cabeza del hombre adulto en ciernes pero con mucha menos potencia que la que tenía cuando era niño) suele aparecer durante la mediavoz 2, pero es normalmente muy difícil de emitir hasta que se alcanza la etapa de nuevo barítono. La SFF se sitúa entre dos y cuatro semitonos por encima del LTP durante las etapas de la muda de la voz masculina. Por lo tanto, la SFF tiende a estar en la base de la tesitura de la voz o incluso coincidir con la nota más grave de la tesitura vocal. Esto significa que durante la adolescencia la voz hablada se encuentra próxima a la voz cantada en la parte baja del registro. Más tarde, en la edad adulta, ambas se separan y la voz cantada extiende ampliamente su registro por encima del SFF y también por debajo (en la voz adulta el SFF se sitúa normalmente entre cuatro y seis semitonos por encima del LTP).

\section{La muda de la voz femenina}

La voz femenina adolescente tiende a un timbre de mayor calidez y opacidad que la de un coro infantil. No es realmente soprano ni alto, sino que todas por regla general parten de un registro medio de mezzo-soprano o soprano II (GACKLE, 1985). Todas las mujeres durante la muda de la voz deberían poder intercambiar sus partes vocales de modo regular de tal manera que tengan la oportunidad de cantar todas las voces femeninas tan pronto como las tesituras sean lo suficientemente cómodas (HARRIS, 1987). También es recomendable vocalizar a todas por igual a lo largo de todo el ámbito de mezzosoprano. El primer signo de muda vocal en la mujer es la aparición de cierta aspereza y exceso de aire en la voz cantada (GACKLE, 1985). Comparado con el sonido aflautado de la niñez, este sonido nuevo áspero y soplado puede parecer desagradable. Una buena técnica vocal puede tender a minimizar la calidad soplada y áspera de la voz femenina adolescente, pero no es posible eliminar totalmente la falta de aire que sufren las chicas en esta edad al cantar, ya que esto solo se resuelve en la medida en que la musculatura laríngea madura y es capaz de cerrar la glotis convenientemente.

Como introducción a este tema, se puede decir que el signo más representativo de la muda de la voz femenina (GACKLE, 1991) es la aparición de ciertas características de disfonía transitoria, las cuales se suelen manifestar a través de uno o varios de los siguientes síntomas disfónicos (cuya aparición se debe al normal desarrollo de la laringe femenina adolescente y no significan por sí solos la existencia de ninguna patología):

a) Imprecisión e inseguridad para cantar afinadamente.

b) Desarrollo de algunos pequeños intervalos de desaparición de la voz a lo largo del registro o ámbito. 
c) Aparición de la voz ronca en la emisión.

d) Ruptura de la voz ocasional durante el canto produciendo saltos de registro.

e) Aspereza en el color de la voz.

f) Emisión incómoda y dificultades en la fonación.

g) Aparición de posibles cambios transitorios en la fonación durante los ciclos menstruales.

h) Descenso de los registros de SFF de la voz hablada.

i) Incremento notable de un exceso de aire en la voz como regla general.

j) El LTP de la voz desciende gradualmente una tercera a lo largo de la adolescencia.

k) El HTP desciende ligeramente a lo largo de la adolescencia (una segunda), pero en muchos casos permanece durante la muda como un registro agudo aislado, de poca calidad y con pocas posibilidades reales de ser usado para cantar, (ya que la tesitura cantable suele estar descendida muy por debajo del HTP).

l) El ámbito permanece estable en la zona de mezzo-soprano en términos generales.

m) Reducción e inconsistencia de las tesituras cantables que por lo general descienden en la etapa más crítica de la muda (post-menarquia), durante la cual fluctúan considerablemente, presentando una irregularidad considerable de individuo a otro.

Todos estos rasgos vocales que se acaban de citar suelen aparecer con los primeros cambios fisiológicos pre-menárquicos hacia los doce o trece años, (abultamiento del pecho, aparición del vello púbico, ensanchamiento de caderas, etc.). En la actualidad se observa que esta etapa pre-adolescente se adelanta más y más y que la aparición de la menarquia se ha adelantado una media de tres o cuatro meses por década. Por otra parte, la mayoría de estas características vocales tienden a permanecer durante la adolescencia post-menárquica debido a que la laringe femenina crece más a lo ancho que a lo largo y también por la incapacidad fisiológica de la glotis para cerrar convenientemente las cuerdas (АвітвоL, АвітвоL у АвітвоL, 1999). Posteriormente, todas estas características de la voz femenina tenderán a desaparecer gradualmente entre uno y dos años después de la aparición de la menarquia (hacia los catorce o quince años). Digamos que la voz femenina se normaliza cuando la menstruación y todos los signos sexuales secundarios se han estabilizado y desarrollado plenamente en el cuerpo, el cual alcanza en este punto un aspecto más juvenil y maduro (MEURER, GARCEZ, VON EYE CORLETA y CAPP, 2007).

Las fases evolutivas de la voz femenina fueron definitivamente establecidos por GACKLE en 1991, tras un primer intento que realizó en 1985. Esta segunda clasificación de 1991 es la que se describe en este artículo. Antes de nada conviene aclarar que dichas fases, vistas en su conjunto, describen una serie gradual de cambios cualitativos en la voz y por lo tanto, todas ellas se pueden contemplar como distintos matices de un mismo proceso secuencial y gradual que transcurre a lo largo de la adolescencia. Las diferencias entre cada una de ellas dependen fundamentalmente del estado madurativo de la laringe, del timbre de la voz y de la calidad en la fonación. Por otra parte, las edades de cada fase tienen un mero carácter orientativo y no deben entenderse como algo definitivo. A continuación se muestran las características principales de cada una de ellas:

a) Fase I (pre-adolescencia): entre los ocho nueve años y los once. Puede llegar hasta los doce dependiendo del ritmo de desarrollo hormonal (AFF: Do4-Re4). La voz cantada posee aún un sonido aflautado, propio de la voz infantil, y su sonoridad es similar a la de soprano, similar a la voz del niño (pero más ligera y con menos volumen que la de aquel)

b) Fase II-A (adolescencia/pre-menarquia): entre los once o doce años, aunque puede alargarse hasta los trece. Es la época que precede a la aparición de la primera menstruación (menarquia) cuando las características sexuales secundarias comienzan a aparecer (desarrollo del pecho, anchura de caderas, vello púbico, etc.), afectando todo ello también a la laringe y a la voz. Los primeros síntomas de la muda de la voz se manifiestan (AFF: Si3-Do\#4). La voz cantada presenta una tendencia hacia un sonido soplado, con exceso de aire (sobre todo en el agudo), debido a la aparición de la hendidura transitoria que impide cerrar convenientemente la glotis. Puede aparecer una ruptura de registro o salto (que separa los registros de cabeza y pecho) entre Sol4Si4. En algunos casos se han descrito problemas en la fonación en el registro de pecho en torno 
al Do4 (algunas chicas parecen tener problemas con el registro grave en esta etapa y presentan una aparente pérdida transitoria de graves). Características de esta etapa:

- incomodidad al cantar;

- poca potencia sonora, dificultades para cantar fuerte;

- voz muy aireada en el registro de cabeza;

En la mayoría de los casos, aparece un sonido lleno en el registro de pecho que contrasta muy obviamente con el timbre infantil, pobre y aireado de la voz de cabeza. Hay un evidente salto entre ambos registros.

c) Fase II-B (adolescencia/post-menarquia): entre los trece y catorce años, aunque puede alargarse a los quince según las circunstancias de desarrollo. Comienza con la aparición de las primeras menstruaciones (menarquia) y se extiende en el tiempo durante un año más o menos. Es el momento más impredecible, caótico, crítico y álgido de la muda de la voz femenina (ABITBOL, АвітвоL у АвітвоL, 1999). Representa la cúspide de este proceso (AFF: La3-Do\#4). La voz cantada pasa por un momento crítico que presenta las siguientes características:

- la voz aireada persiste en general (sobre todo en el agudo);

- voz de cabeza constreñida, se pierde la facilidad para emitir con la voz de cabeza, aparecen dificultades en el agudo;

- la tesitura fluctúa arriba o abajo, en ocasiones desciende o asciende a los extremos del ámbito (o muy aguda o muy grave) dejando sólo unas 5-6 notas útiles para cantar;

- el ámbito en general también se acorta, y suele presentar rupturas de registro o saltos entre Sol4-Si4 y a veces también entre Re5-Fa\#5;

- $\quad$ el timbre y el color de la voz se vuelve más sucio y rasgado (falta de claridad en el sonido);

- aparece la ilusión de ser una voz contra-alto porque este registro es el que menos sufre en esta etapa; aunque cantar de pecho puede ser más fácil en este periodo, cantar sólo en el grave puede sobrecargar y dañar la voz;

- la voz presenta ronquera aunque no se esté resfriada;

- frecuentes rupturas de voz;

- incomodidad al cantar.

En esta etapa es preciso seguir vocalizando la voz en todo el ámbito a pesar de las dificultades que aparecen en la fonación (pero sin llegar a dañar la voz). Para monitorizar cada caso adecuadamente, es preciso realizar audiciones periódicas a las alumnas en esta etapa.

d) Fase III (voz juvenil femenina): se suele alcanzar esta etapa sobre los catorce o quince años, en algunos casos se demora hasta los dieciséis (AFF: Sol\#3-Si3). El ámbito vocal se extiende de nuevo en esta etapa. Aquellas voces que poseen una mayor calidad presentan normalmente un amplio ámbito, también en el grave, pero, cuidado, no por ello son altos (que lleguen a las notas graves no significa que su tesitura sea ésa.)

Los registros de cabeza y pecho tienden a fusionarse. Todavía persiste una ruptura de registro o salto en la zona que coincide con las notas del pasaje de la voz femenina adulta (Re5-Fa\#5). Las características de esta etapa son:

- el color del sonido es más opaco, profundo y lleno que el de la etapa anterior;

- aparece el vibrato, la voz gana en resonancia, volumen y agilidad;

- el triángulo morfológico tiende a cerrarse, la glotis comienza a cerrarse bien;

- la claridad vuelve a la voz y poco a poco se gana un color del sonido más rico y lleno;

- el timbre se parece progresivamente cada vez más al de una mujer adulta. 
A continuación podemos observar un esquema musical de las fases vocales de GACKLE (1991) anteriormente descritas ${ }^{1}$ :

Fase I: $\quad$ Fase II-A: $\quad$ Fase II-B: $\quad$ Fase III:

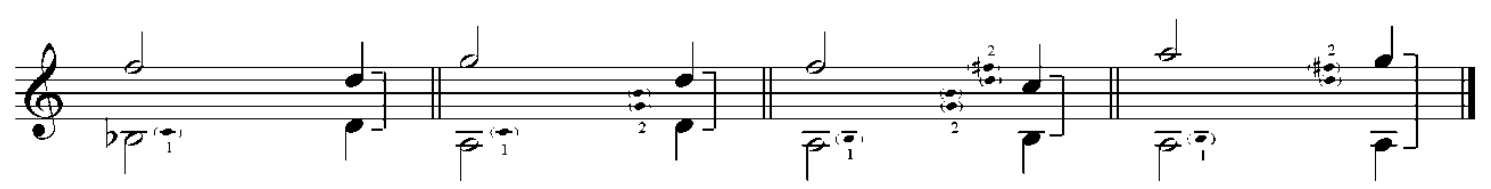

La tabla siguiente sintetiza las características de las fases II-A, II-B y III (GACKLE, 1991):

\begin{tabular}{|c|c|c|}
\hline $\begin{array}{l}\text { II-A. pre-menarquia: primeros } \\
\text { "síntomas" }\end{array}$ & II-B. post-menarquia: etapa crítica & $\begin{array}{l}\text { III. voz juvenil: recuperación } \\
\text { gradual }\end{array}$ \\
\hline $\begin{array}{l}\text { ruptura en el color de la voz } \\
\text { muy evidente entre cabeza y } \\
\text { pecho } \\
\text { timbre infantil, pobre y airea- } \\
\text { do de la voz de cabeza } \\
\text { - } \\
\text { sonido lleno en el registro } \\
\text { medio-grave, de pecho. } \\
\text { poca potencia sonora }\end{array}$ & $\begin{array}{ll}\text { - } & \text { la voz sigue aireada y el ámbito se re- } \\
\text { - } & \text { duce } \\
\text { voz de cabeza apretada, dificultades en } \\
\text { - } & \text { el agudo } \\
\text { - } & \text { resituras fluctuantes y reducidas de voz, incomodidad al cantar. } \\
\text { - } & \text { voz sucia, poco clara } \\
\text { - } & \text { falsa apariencia de contralto } \\
\text { - } & \text { voz ronca y áspera en ocasiones }\end{array}$ & $\begin{array}{ll}\text { - } & \text { color del sonido más opaco y } \\
\text { - } & \text { cálido } \\
\text { - } & \text { voz gana en claridad } \\
\text { - } & \text { exceso de aire desaparece } \\
\text { - } & \text { ámbito y tesitura más amplios }\end{array}$ \\
\hline
\end{tabular}

\section{DISEÑO DEL ESTUDIO}

Este estudio fue realizado en un instituto de enseñanza secundaria de la zona sur de Madrid, durante el curso académico 2008-9 con un total de treinta participantes, todos ellos alumnos del instituto y miembros del coro de alumnos del centro, con el cual el investigador mantiene una vinculación docente directa. En este coro no hay una prueba selectiva de acceso, sino que todo el que lo desea puede participar en él, independientemente de la calidad vocal de cada alumno o alumna. Los alumnos que presentan problemas de afinación tienen un ensayo extra a la semana sólo para ellos, con la finalidad de que reciban un apoyo que les vaya posibilitando poco a poco la participación satisfactoria en el grupo junto con los demás.

Este estudio parte de la adopción de un rol de profesor-investigador durante toda la investigación (ROBERTS, 1994), el cual consistió en alternar ciclos de planificación docente con ciclos de investigación y con ciclos de acción docente, de enseñanza. Esta estrategia de docente-investigador se fundamentó en la necesidad de mejorar la práctica docente (WHITEHEAD, 1989; CAIN, 2008) en relación con el desarrollo de la voz de los adolescentes que formaban parte del coro del instituto. El punto de partida fue una pregunta de carácter reflexivo que en este caso se materializó en “¿Cómo mejoro la eficacia de los ensayos al frente del coro? Esto llevó, en primer lugar, a una constante evaluación y a un proceso de transformación de la enseñanza dentro de una perspectiva eminentemente interpretativa, naturalista. Dentro de este contexto de investigación, se realizaron numerosas entrevistas enmarcadas en una metodología cualitativa, con el objeto de comprender los aspectos psicológicos del canto escolar tal y como lo interpretaban los adolescentes. Esta parte de la investigación dio resultado a un estudio con cuerpo propio, el cual será eventualmente publicado en un futuro si las circunstancias lo permiten.

Sin embargo, a partir de los resultados de las entrevistas (donde los alumnos manifestaban su preocupación por conocer con seguridad el estado madurativo de su voz para poderse identificar

\footnotetext{
${ }^{1}$ Las blancas indican el ámbito vocal y la nota entre paréntesis señalada con (1) indica el SFF aproximado; las negras unidas con corchetes indican la tesitura cantable, y las dos notas entre paréntesis señaladas con (2) indican el intervalo donde es más probable que aparezca una ruptura o salto de registro.
} 
con ella de manera inequívoca) se volvió evidente en un momento de la investigación, la necesidad de desarrollar una metodología eficaz que permitiera clasificar adecuadamente la voz adolescente de ambos sexos. El coro del instituto necesitaba de un tratamiento específico en relación a la muda de la voz y en relación a los datos obtenidos a través de la investigación cualitativa, los cuales versaban principalmente sobre el modo en el cual los alumnos de ambos sexos interpretaban su práctica coral dentro del contexto escolar en relación a la construcción de sus identidades de género.

Sin tener que abandonar el paradigma interpretativo que enmarcaba toda la investigación, se vio por lo tanto, la necesidad de estudiar los aspectos fisiológicos y madurativos de la voz aplicando una metodología cuantitativa a través de la realización de un estudio de fonación que permitiera clasificar la voz de los adolescentes, estudio del cual se ocupa este artículo. Los resultados de este estudio de fonación permitieron comprender mejor las características vocales de los alumnos del coro, y en función de las mismas, elegir más adecuadamente el repertorio y planificar más eficazmente los ensayos, permitiendo así que la línea vocal de cada parte coral pudiera estar al alcance de cada alumno según el estado madurativo de su voz. Tuvo como resultado que los alumnos pudieron ser clasificados de un modo correcto según el estado madurativo de su voz, lo cual les permitió identificarse mejor con su voz y generar una identidad vocal más clara e inequívoca. Todo esto tuvo resultados positivos para la parte cualitativa de la investigación, de tal modo que ambas metodologías se complementaron dentro del marco del rol de profesor-investigador que enmarcó toda la investigación en su totalidad.

El estudio de fonación que se presenta aquí tuvo en los alumnos del coro a los principales participantes del mismo. Entre todos los miembros del coro, que en total sumaban cuarenta y cinco en el momento de la realización de esta investigación, treinta de ellos participaron en un estudio de fonación consistente en la realización de una prueba vocal con el objetivo de clasificar sus voces según la taxonomía de COOKSEY (1992) y GACKLE (1985) para la voz adolescente masculina y femenina, respectivamente. Estos alumnos fueron escogidos al azar siguiendo un criterio paritario: tres alumnos por edad y sexo entre doce y dieciséis años. La muestra fue lo más amplia posible de entre los alumnos del coro sin tener que abandonar dichos criterios. Si el método de clasificación vocal propuesto en el estudio demostraba su validez, éste sería extensible al resto de los alumnos del coro como herramienta eficaz para clasificar sus voces.

Se debe destacar que las teorías de COOKSEY (1992) y GACKLE (1985), si bien ofrecen un marco teórico claro sobre la voz adolescente, carecen de un método de clasificación vocal que pueda ser aplicado de un modo universal. En sus escritos no se encuentra ninguna referencia a este respecto, dejando este asunto de la metodología de la clasificación vocal al arbitrio del lector. Este hecho explica que los estudios previos existentes en este terreno pertenezcan mayormente al ámbito logopédico, y por lo tanto, la investigación educativa en este terreno ha venido marcada en las últimas décadas por una búsqueda de las variables fisiológicas que influyen en el desarrollo de la voz durante la adolescencia. Así ocurre con varios estudios de fonación (BARLOW y HowARD, 2002, 2005, 2007) que presentan un diseño similar al estudio de este artículo, consistente básicamente en realizar una única prueba vocal sistemática a una determinada muestra de población adolescente en un momento puntual de su desarrollo madurativo. Otros estudios de fonación consultados (FuCHS et al., 2001; FUCHS et al., 2008) se acercan más a un diseño longitudinal, donde la muestra es sometida a una prueba de voz en distintos momentos durante una determinada franja temporal, con el objeto de observar su evolución.

La metodología empleada en este estudio ha sido, como ya se ha dicho, la cuantitativa y el principal método de recogida de datos ha consistido en la realización de una prueba vocal grabada, la cual ha sido diseñada por el investigador. Los datos que se registraron a partir de la misma provienen de la investigación vocal en el ámbito anteriormente citado, y tanto COOKSEY (1993) como GACKLE (1991) ya citaron en sus escritos algunas de estas variables (las resaltadas en negrita, ya que el resto han sido diseñadas por el investigador para este estudio) pero sin aportar una metodo- 
logía universal de obtención de las mismas que sea aplicable en la educación escolar. Dichas variables son las siguientes:

- Afinación media de la voz hablada (también llamado SFF, ya que es el sonido a partir del cual se suele comenzar a hablar/recitar con naturalidad): es la afinación habitual de la voz hablada de un individuo (el AFF es otra variable que a veces se utiliza igualmente para medir en $\mathrm{Hz}$ la tesitura de la voz hablada).

- Nota de recitación aguda: se identifica en el estudio bajo las siglas NRA. Se trata de un dato que finalmente no va a ser muy relevante, pero que en un principio resulta fundamental para comparar el dato obtenido a partir de la SFF. Es la nota más aguda de la voz hablada que haya sido empleada durante el recitado del poema. Se sitúa por encima del SFF, en la zona media de la tesitura cantable. Se localiza en las inflexiones agudas de la voz hablada, dependiendo del estilo declamativo del individuo. Suele estar próximo al límite agudo de la tesitura de la voz hablada.

- Nota más grave de la tesitura cantable: se identifica en el estudio bajo las siglas NGT. Suele situarse próxima al SFF.

- Nota más aguda de la tesitura cantable: se identifica en el estudio bajo las siglas NAT.

- Nota aguda límite del registro o ámbito: HTP (high terminal pitch).

- Nota aguda límite del registro o ámbito: LTP (low terminal pitch). Se sitúa ligeramente por encima del límite inferior de la tesitura de la voz hablada.

Además, COOKSEY (1993) y GACKLE (1991) citan en sus estudios los diversos problemas de emisión vocal asociados al desarrollo madurativo de la adolescencia, por lo cual parecía recomendable tener este aspecto en cuenta en el presente estudio. Por lo tanto, también se fueron registrando los datos más relevantes en torno a las dificultades presentadas en relación a la fonación. Estas variables quedaron registradas a lo largo de la prueba por las anotaciones individuales realizadas in situ por las apreciaciones del investigador, donde quedaban reflejadas las dificultades observadas concernientes a los aspectos cualitativos de la fonación de cada alumno. Las variables relacionadas con los problemas de emisión vocal han sido fundamentalmente las dos siguientes:

- Dificultades para cantar afinadamente, los cuales han sido descritos por COOKSEY (1993) como prioritariamente asociados a la voz masculina adolescente y atribuida a la desorientación auditiva que los chicos sufren cuando su registro desciende.

- Aparición de una voz aireada en exceso (con dificultades transitorias para cantar en el registro de cabeza), la cual ha sido descrita por GACKLE (1991) como prioritariamente asociada a la voz femenina adolescente, como consecuencia de una falta de madurez laríngea que impide cerrar convenientemente la glotis durante algún tiempo en la pubertad.

\section{Metodología del estudio de fonación:}

Los datos se registraron según el sistema de índices acústicos de banda audible ${ }^{2}$ :

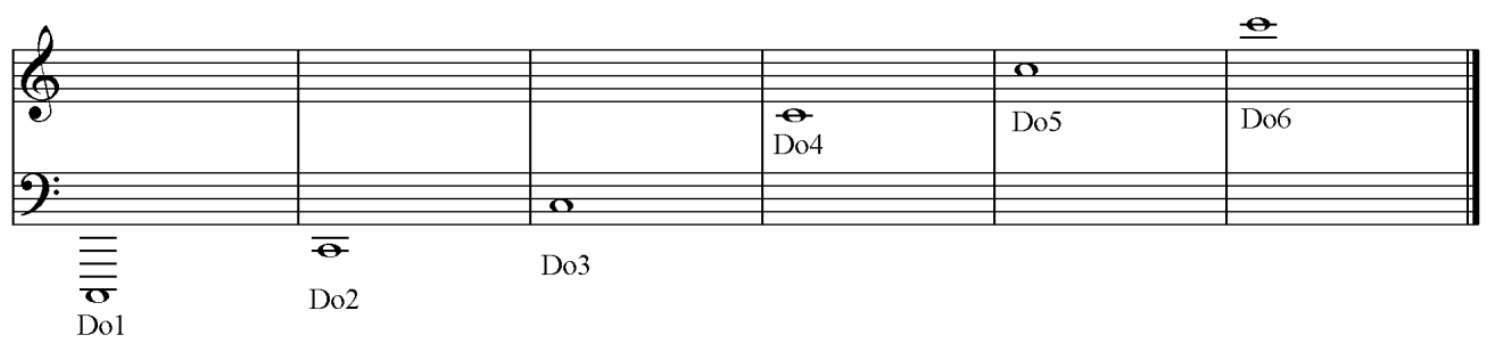

\footnotetext{
${ }^{2}$ En este sistema los números indican la cantidad de octavas en que se encuentra un sonido dado con respecto al umbral de audibilidad. Así, el Do4 (Do central del piano) indica que se trata de un Do cuatro octavas por encima del umbral.
} 
Cada una de las pruebas fue grabada sonoramente. Para ello se contó con una grabadora digital situada en el lugar de la prueba. Los datos de cada variable se registraban in situ por el investigador, contrastando la afinación de la emisión vocal de cada alumno con la afinación del piano electrónico con el que se realizaba la prueba, si bien los datos eran de nuevo corroborados a posteriori usando un software musical especializado, (contrastando los registros sonoros con un afinador digital) a través del cual se terminaban de ubicar las frecuencias con precisión. Cada alumno realizaba la prueba íntegramente en una sola sesión (con una duración aproximada entre seis y ocho minutos) donde sólo estaba presente el alumno concernido y el investigador. Se contemplaron varias sesiones de grabación para ir escuchando a cada alumno participante por turnos establecidos durante los recreos del horario escolar. En cada turno participaban dos alumnos, el que esperaba turno lo hacía fuera, en otra sala contigua. Entre la primera sesión y la última transcurrieron tres semanas situadas entre los meses de febrero y marzo de 2009. Los pasos a seguir durante el desarrollo de cada prueba eran los siguientes:

1) Charla breve introductoria con el alumno.

2) Recitado del poema. Registro de la NRA.

3) Contar desde veinte hasta uno. Registro de la SFF.

4) Ejecución vocal de una melodía en una afinación elegida por el alumno o bien a partir de la SFF. Registro de la AFF y de posibles dificultades de fonación.

5) Ejecución vocal de la melodía anterior transportada ascendente y descendentemente, seguida de la vocalización $\mathrm{n}^{\circ} 1$. Registro de la NAT y la NGT. Corroboración de los eventuales problemas en la emisión vocal.

6) Ejecución vocal de la vocalización $n^{0} 2$. Registro del HTP.

7) Ejecución vocal de la vocalización $n^{0} 3$. Registro del LTP.

La prueba comenzaba con una breve e informal charla con cada participante, ya que en muchos casos sirvió para tener una primera impresión de la afinación aproximada habitual de la voz hablada. A continuación cada alumno recitó dos veces un fragmento de un poema, tras lo cual fue posible anotar la nota más aguda y la más grave del recitado. La nota de comienzo de la recitación solía coincidir con el SFF, ya que es el sonido a partir del cual se suele comenzar a hablar/recitar con naturalidad. La nota de recitación aguda (NRA) estaba situada por lo general, entre dos y cinco semitonos ascendentes. En este punto de la prueba ya se habían obtenido estos dos datos de la voz hablada, correspondientes a las notas entre paréntesis (el ejemplo corresponde a los datos de un varón catalogado como mediavoz 1).

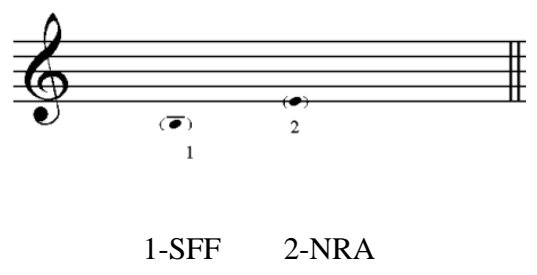

Seguidamente, los alumnos contaban desde veinte hasta uno, hacia atrás, manteniendo una recitación lo más uniforme posible, evitando que se entrecortara. La afinación de este recitado se encontraba (en la práctica totalidad de los casos) cerca de los datos del SFF obtenidos anteriormente, de tal modo que los mismos quedaban confirmados. A continuación, los alumnos cantaban la canción "Cumpleaños feliz" sin ninguna referencia previa de afinación desde el teclado. Por ejemplo, cualquier varón que se encontraba al comienzo del proceso de la muda de la voz podía comenzar a cantar de modo intuitivo en torno al Sib3, una nota muy cercana al SFF de los chicos catalogados como mediavoz 1: 


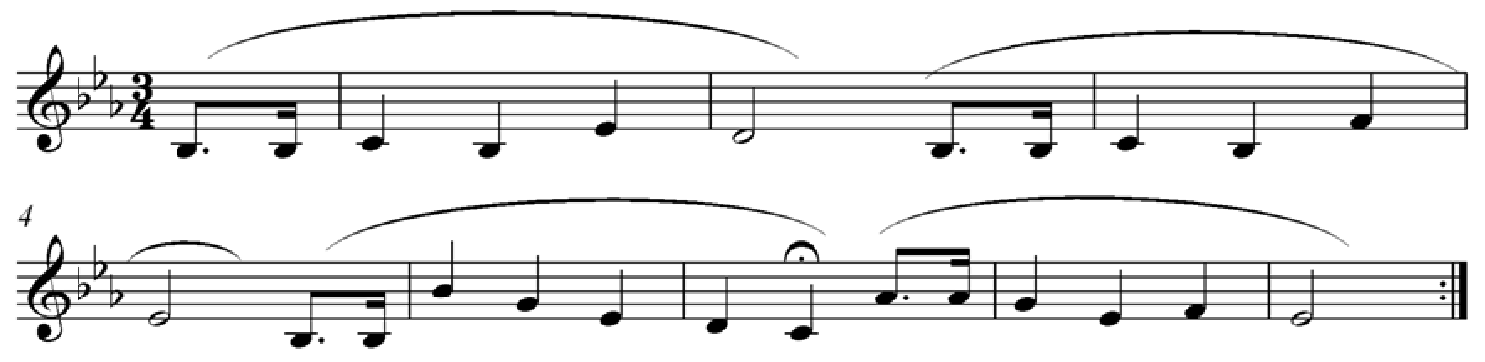

En algunos casos, la tercera frase de esta melodía suponía una dificultad que no era superada satisfactoriamente, sobre todo para las mujeres (MADDOX, 1986). Para ellas, una entonación correcta de esta frase suponía un cambio repentino de la voz de pecho a la de cabeza que no siempre se resolvía bien, y en consecuencia algunas alumnas desafinaban inicialmente. Como el objetivo de esta primera fase de la prueba era delimitar exclusivamente el registro cantado de pecho, en ocasiones se les pedía que cantaran sólo la primera frase de "Cumpleaños feliz" a partir de la SFF anotada a partir de los datos anteriores (recitado literario y numérico). De este modo, el resultado vocal era en todos los casos satisfactorio. Todos los alumnos pudieron cantar y emitir afinadamente cerca del límite grave de su voz de pecho, el cual se encuentra muy próximo a la voz hablada en la adolescencia.

Para confirmar este dato los alumnos también cantaban a partir de la misma nota (la SFF era siempre la nota inicial) algunas de estas otras dos canciones: "Jingle bells" (sólo el estribillo, en español) o "A por ellos” (la famosa canción de la selección española de fútbol). Normalmente los chicos se decantaban por la segunda y las chicas por la primera. En este preciso momento, y de acuerdo con los datos de recitación y la afinación utilizados por cada alumno hasta este momento, se procedía a asignarle un registro provisional a cada candidato. Una vez asignado el registro de la voz hablada y de la voz cantada de pecho, ahora se trataba de hallar la tesitura cantable de cada individuo. Para ello, los alumnos cantaban la melodía de cumpleaños feliz (o cualquiera de las otras dos canciones) a veces con distintas vocales, acompañada al piano por la armonía y doblando la melodía. Se realizaban los transportes ascendentes o descendentes necesarios (tocando un acorde de tónica antes de comenzar) hasta ubicar correctamente la tesitura cantable del individuo. Para confirmar que la ubicación de la tesitura era correcta, cada alumno cantaba la vocalización $\mathrm{n}^{\circ} 1$ (con las sílabas “si”, “no”) en la afinación correspondiente con la etapa vocal pre-asignada (comenzando por la NGT y realizando un transporte de hasta cuatro semitonos ascendentes):

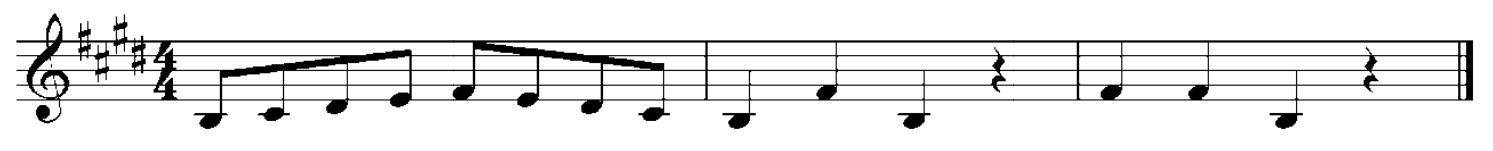

La emisión en cada vocalización debía ser natural, no forzada, sin dificultades y con un buen grado de armónicos naturales (FREER, 2009b). Llegados a este punto, se habían obtenidos estos dos datos nuevos, correspondientes a las notas abarcadas por el corchete (la inferior es la NGT y la superior la NAT).

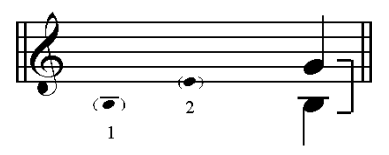

Seguidamente se continuaba con la vocalización $n^{\circ} 2$ al piano, que el alumno repetía cantando: 


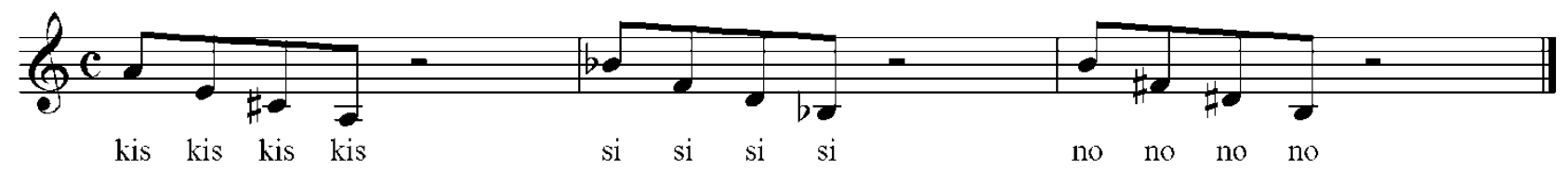

El objetivo era conseguir que el alumno se esforzara en alcanzar los extremos de su emisión vocal pero sin forzar ni dañar su laringe. Para ello se utilizaba esta vocalización enérgica pero ligera y rápida, en arpegio descendente desde el agudo. Se comenzaba por la NAT y se realizaban los transportes necesarios (se tocará un acorde de tónica antes de comenzar) hasta alcanzar el límite agudo (HTP) del registro o ámbito, correspondiente con la nota blanca superior.

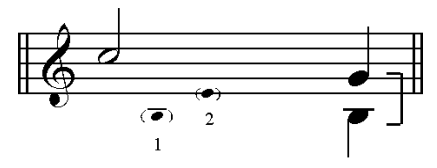

Ahora tendríamos ya localizados los límites del registro o ámbito. Si se consideraba oportuno, también era posible confirmar que habíamos alcanzado el HTP utilizando la vocalización n³ (comenzando por una cuarta ascendente desde la NGT y realizando los transportes ascendentes necesarios).

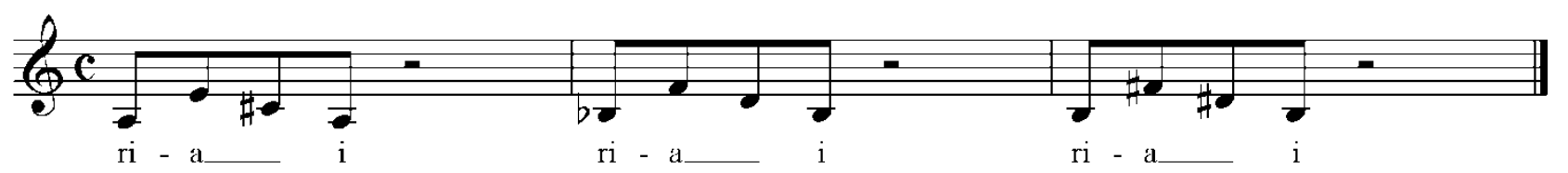

Para finalizar, se volvía a usar la vocalización $\mathrm{n}^{0} 1$ en sentido descendente para hallar el LTP, correspondiente con la nota blanca inferior. (El LTP suele estar ligeramente por encima del límite grave de la tesitura de la voz hablada). En este punto ya se tenían localizados los límites del registro o ámbito vocal (correspondientes con las notas blancas):

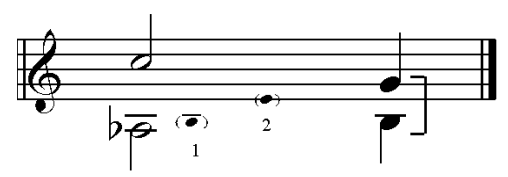

De este modo, habríamos completado todos los datos de la ficha que constituyen las principales variables del estudio (en el ejemplo, correspondientes a un varón catalogado como mediavoz 1).

\section{Análisis de datos}

Este primer gráfico nos deja ver que la evolución de la muestra es bastante estable en las dos primeras fases del desarrollo laríngeo, mientras que a partir de mediavoz 2a aumenta cuantitativamente. Estos datos parecen apuntar que las dos primeras fases de la muda de la voz masculina son estables a la vez que transcurren con cierta celeridad. Una vez que la muda de la voz comienza a asentarse en el registro grave, el ritmo del proceso evolutivo laríngeo se atenúa, decrece y hay por lo tanto más casos de varones clasificados en las últimas etapas. 
Gráfico 1: Evolución por clasificación vocal de la voz masculina adolescente del total de la muestra.

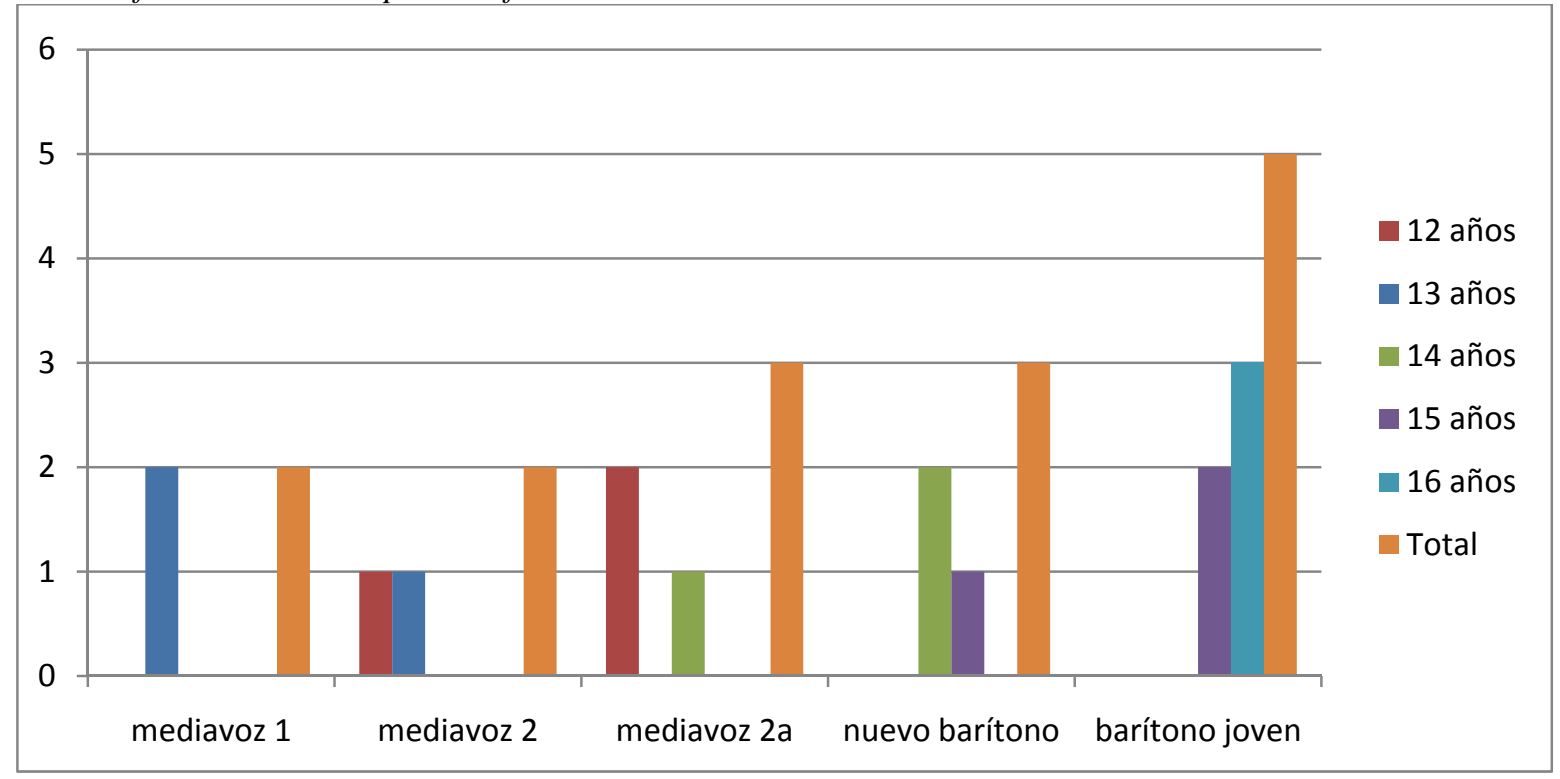

Este segundo gráfico nos presenta los mismos datos clasificados por edades:

Gráfico 2: Evolución por edades de la voz masculina adolescente del total de la muestra.

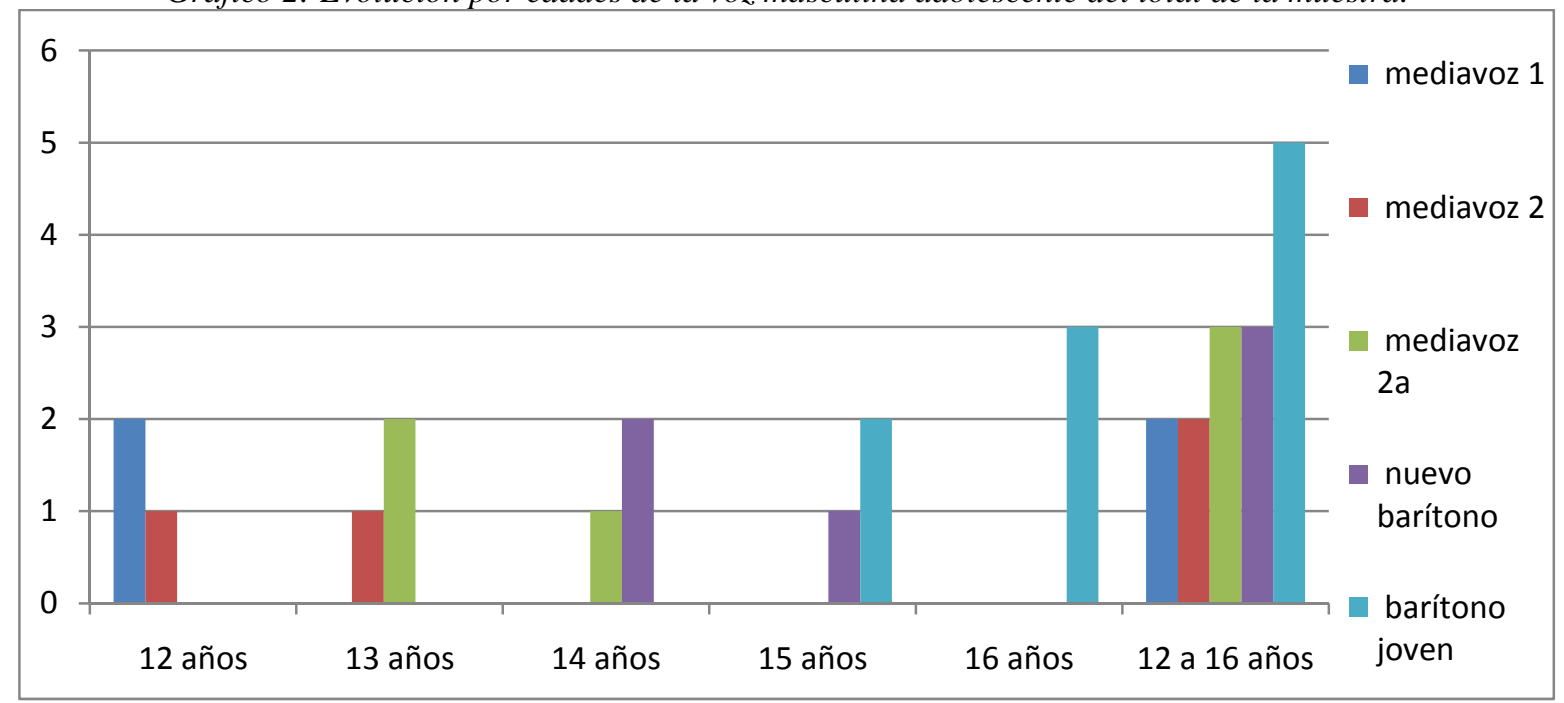

Este gráfico nos permite corroborar los datos obtenidos anteriormente. De nuevo se observa claramente como las primeras etapas de la muda de la voz masculina se presentan estables en relación a la muestra, mientras que a partir de mediavoz 2a aumentan cuantitativamente. También nos deja ver que la muda de la voz se produce fundamentalmente entre los doce y trece años. A partir de los catorce años, las etapas predominantes son las de nuevo barítono y barítono hacia la voz adulta, las cuales marcan el final de todo el proceso. A continuación observamos la gráfica en relación a la voz femenina. 
Gráfico 3: Evolución por clasificación vocal de la voz femenina adolescente del total de la muestra.

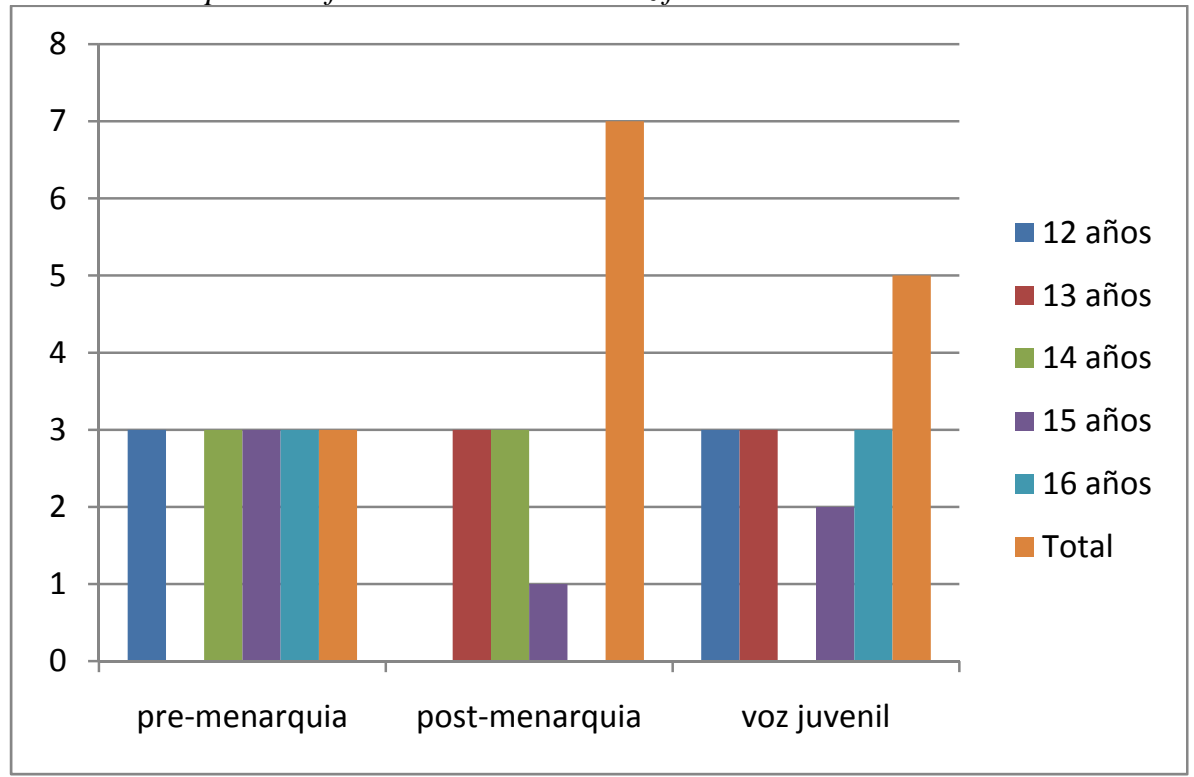

Aquí podemos ver como la etapa posterior a la menarquia es la que más crece, lo cual deja entrever que las dificultades descritas por GACKLE (1985) como propias y características de esta etapa tienden a establecerse en el tiempo de un modo más continuo al que sería deseable. El próximo gráfico de nuevo confirma estos mismos datos de un modo más detallado en torno a los trece y catorce años. Estas edades se revelan como claves para entender cómo el desarrollo madurativo de la laringe femenina parece encallarse durante un par de años, y sólo a partir de los quince comienzan a desaparecer las disfonías transitorias descritas por GACKLE (1985) y por lo tanto, a emerger una voz juvenil femenina, tendente ya hacia la madurez de la voz adulta.

Gráfico 4: Evolución por edades de la voz femenina adolescente del total de la muestra.

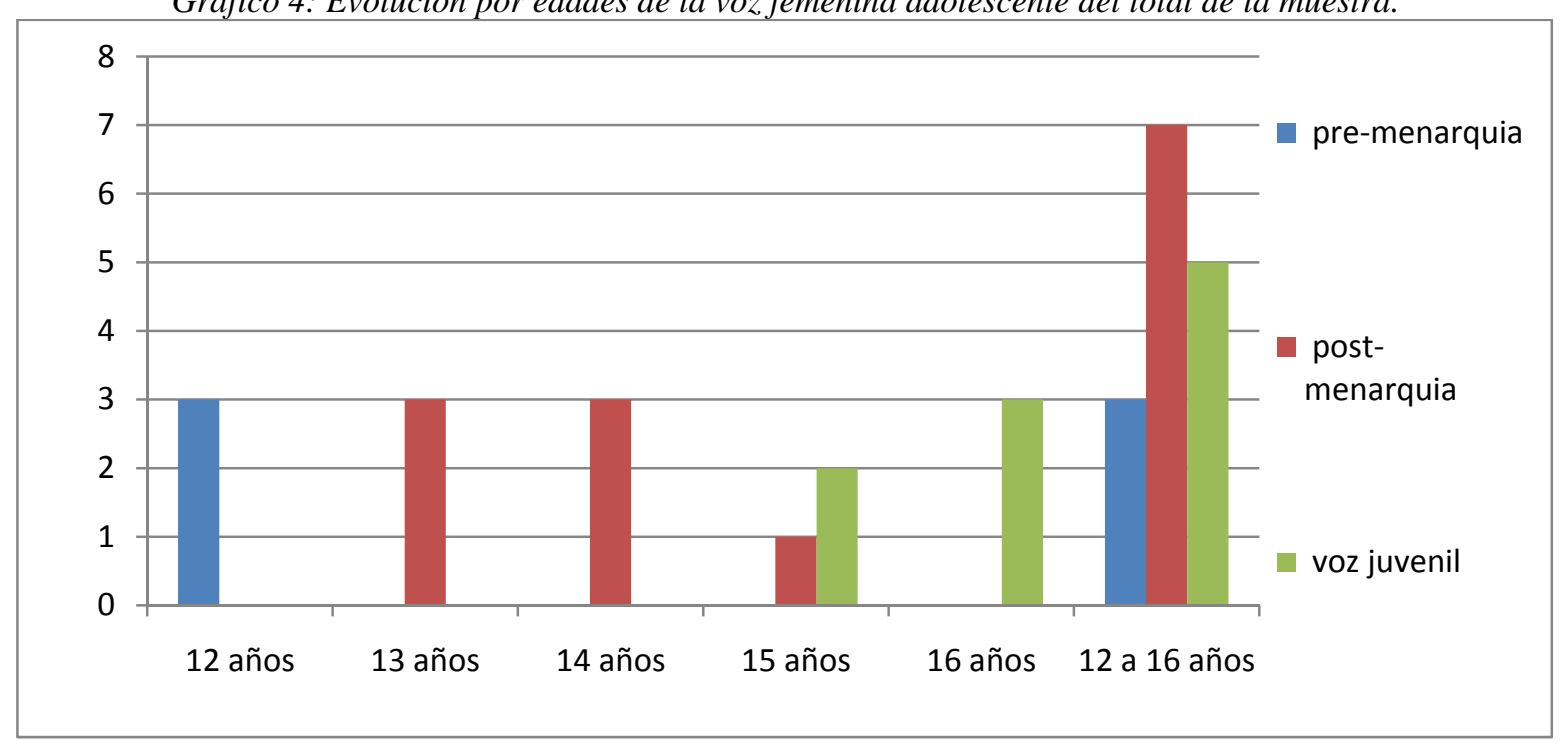

Por último, los datos más relevantes en torno a las dificultades presentadas en relación a la fonación mostraron la aparición de disfonías transitorias asociadas a la maduración laríngea femenina en más del cincuenta por ciento de los casos. 
Gráfico 5: Dificultades en la fonación detectadas por edad del total de la muestra.

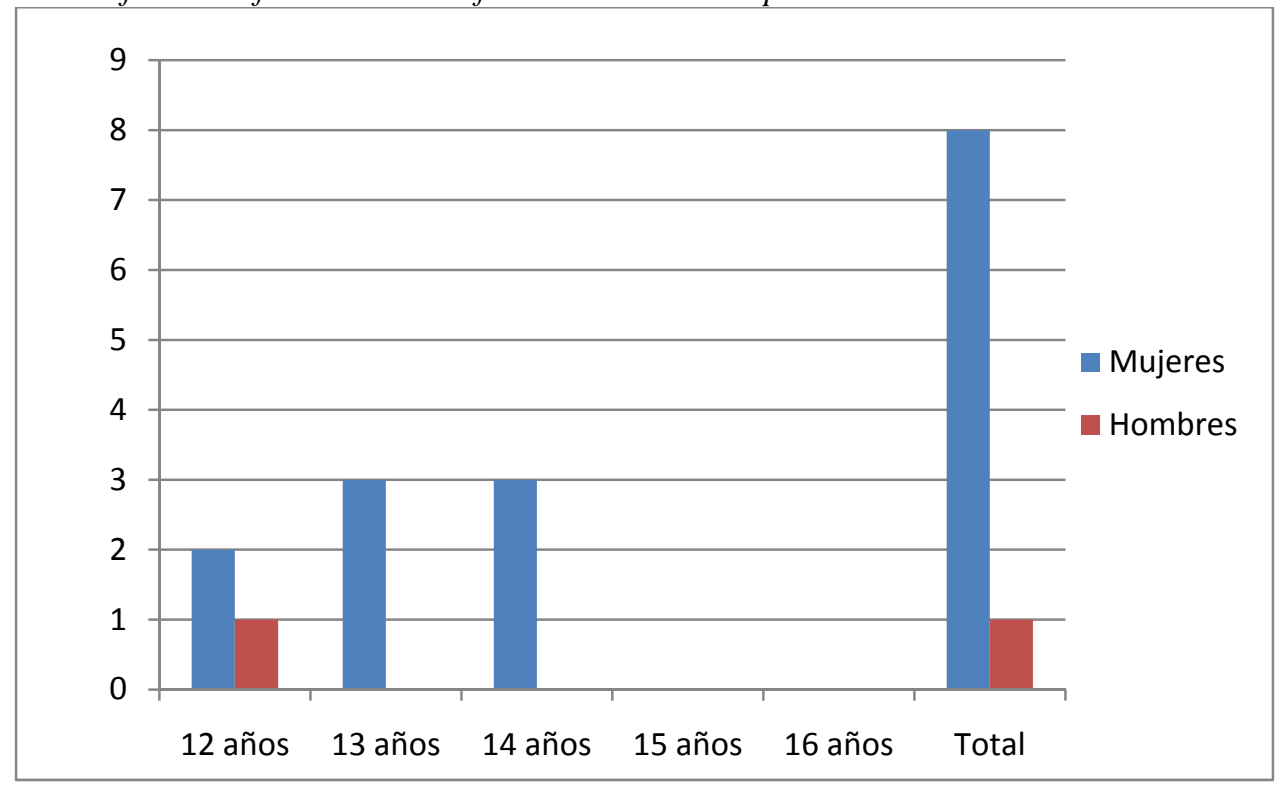

Solamente se apreció el caso de un varón que presentó dificultades en la emisión vocal. Por otra parte, ninguna mujer presento problemas en relación a la afinación de la voz cantada. Por otra parte, con respecto a las dificultades en la afinación detectadas por edades en el total de la muestra, tan sólo dos varones (de un total de quince) mostraron dificultades y en ambos casos, estas aumentaban en la medida en que se ascendía hacia el agudo y la distancia con el registro de la voz hablada (SFF) se hacía más grande. Ambos varones tenían quince años y ya habían prácticamente completado el proceso de muda de la voz, uno fue catalogado como nuevo barítono y el otro poseía una voz más descendida y madura y fue clasificado como barítono hacia la voz adulta. El resto de los varones no presentó dificultad alguna a la hora de afinar.

\section{DISCUSIÓN}

Estudios recientes demuestran que las mujeres adolescentes suelen disponer de una buena autopercepción de su voz (MonKs, 2003) a pesar de sufrir, en la mayoría de los casos, algún tipo de disfonía transitoria (АвIтBOL, AвIтBOL у АвітBOL, 1999). Este dato es confirmado también en este estudio, donde más del cincuenta por ciento de la muestra femenina presentaba dificultades en la fonación. Conviene aclarar aquí que las disfonías femeninas durante la pubertad son absolutamente normales y no deben ser confundidas con las "puberfonías”3 (PUYUELO SANCLEMENTE, 1997:182), las cuales denotan la aparición de una patología de origen endocrinológico en la mayoría de los casos. La muestra de este estudio tiende a confirmar este dato, ya que presenta una concentración femenina en torno a la fase de post-menarquia, alargando así el tiempo durante el cual las chicas tienen dificultades para cantar, y en ningún caso esto hecho se debe a una enfermedad. Por lo tanto, resulta sencillo constatar que las disfonías transitorias que una buena parte de las adolescentes sufren no afectan a las opiniones favorables que ellas suelen tener sobre su voz. No hay estudios internacionales que hablen de la existencia de una identidad vocal negativa imperante entre las adolescentes. Parece ser que las chicas no se dejan influir en su auto-concepto vocal por estas disfonías transitorias, aceptan su voz tal y como es y su actitud hacia el canto es en general positiva. Cabría preguntarse el porqué, lo cual constituye un buen punto de partida para ulteriores investigaciones.

\footnotetext{
${ }^{3}$ Las disfonías asociadas a la adolescencia reciben el nombre de puberfonías únicamente cuando describen trastornos patológicos asociados a la muda de la voz. Suelen presentarse en adolescentes varones cuya laringe no se desarrolla convenientemente y por lo tanto, no consiguen agravar su registro de un modo normal. Esta patología suele estar provocada por alguna endocrinofonía (tirotixicosis, enfermedad de los órganos sexuales) previa.
} 
También llama la atención que en la muestra masculina de este estudio, la velocidad de la muda de la voz es inversamente proporcional al descenso del registro. Cuanto más aguda es la voz, más rápida es la muda y menos duradera, mientras que cuanto más desciende el registro, más se ralentiza el proceso de la muda. Por otra parte son conocidas las dificultades que algunos chicos presentan a la hora de afinar (WELCH, 1979; PRICE, 1994) las cuales algunos autores atribuyen a la desorientación auditiva que ellos sufren cuando su registro desciende, lo cual les dificultaría escucharse a sí mismos correctamente y propiciar la típica actitud del “desafinado” (AGUILAR, 2007) que desaparece normalmente con la edad. Sin embargo, en este estudio tan solo dos chicos presentaron este perfil. Llama igualmente la atención que, a pesar de ello, haya una casuística tan amplia de jóvenes y adolescentes que tienen una mala impresión sobre su voz y piensan que cantar es una tarea ardua y difícil de conseguir (FREER, 2009a). Por lo tanto, pareciera que los varones adolescentes tienden a pensar que cantar es un asunto complicado, aunque los casos de desafinados entre la población masculina de la muestra de este estudio no es ni mucho menos amplia. Cabría, por lo tanto, preguntarse si esta tendencia también se observa en muestras de poblaciones mayores, y en consecuencia, hasta qué punto es cierto la idea preconcebida, que en algunos casos se tiene, acerca de la supuesta incompetencia que se presupone a los varones a la hora de cantar afinadamente durante la adolescencia. Un estudio reciente (DEMOREST y CLEMENTS, 2007) realizado en Seattle (Estados Unidos) con sesenta varones entre once y trece años demostró que era mucho más frecuente encontrar alumnos que cantaban normalmente, y que los alumnos monótonos eran realmente pocos. Este estudio también evidenció que la competencia masculina para cantar afinado dependía mucho más de la experiencia previa relativa al canto de cada individuo que de cualquier otro factor, incluido el descenso gradual de la voz. Parece que el hecho de que la muda de la voz se produzca, no es por sí solo, un factor que predisponga a cantar desafinadamente.

Otro estudio muy reciente al respecto (WILLIS y KENNY, 2008) realizado sobre setenta y nueve varones adolescentes australianos entre trece y catorce años muestra que casi todos ellos fueron capaces de afinar cuartas, quintas y octavas cuando se partía de una nota cercana al SFF de cada participante que se sometió a una prueba de entonación. Es decir, parece ser que cuando se parte de un registro cercano a la voz hablada de cada individuo (según el estado madurativo donde se encuentre), los problemas de afinación se minimizan. Los dos varones que desafinaron en el estudio que presento en este artículo, presentaron la misma tendencia. Su competencia para emitir correctamente era mayor cuanto más grave cantaban y más cerca se encontraban de su voz hablada (SFF). Todo esto tiene sentido si consideramos la teoría de COOKSEY (1977a, 1977b, 1977c, 1978) que relaciona íntimamente la voz hablada con el registro grave de cada alumno varón durante la adolescencia. Esta unión durante los años de la muda de la voz es muy estrecha y se muestra como una de las claves a considerar si queremos tener éxito a la hora de enseñar a cantar a los adolescentes que se ven inmersos en el proceso de la muda de la voz masculina.

\section{IMPLICACIONES EDUCATIVAS}

El desconocimiento por parte de los alumnos sobre el proceso de la muda de la voz es el campo de cultivo ideal para que los prejuicios imperantes sobre el canto coral en la educación secundaria sigan vivos por muchos años entre alumnos y profesores. Por lo tanto, si deseamos comenzar a operar un cambio entre nuestros alumnos a este respecto, es manifiesto que deberíamos comenzar por un iniciar un programa vocal específico para la educación secundaria que comprendiera, como mínimo, los siguientes puntos:

a) Explicación teórica general de la muda de la voz, tanto masculina como femenina (ELORRIAGA, 2010a, 2010b). Poner especial énfasis en la construcción de un discurso que fomente la idea de que todos pueden encontrar su voz, de que cada voz es única y personal, evoluciona a un ritmo distinto, y que por lo tanto, todos y cada uno de los alumnos pueden aprender a cantar si se lo proponen, con la ayuda y guía docente necesaria. 
b) Iniciar un proceso de entrenamiento vocal basado en el movimiento corporal y la ejecución de ejercicios de coordinación psicomotriz como base fisiológica para la adquisición de una técnica vocal básica entre los adolescentes (Phillips, 1996; PHILlips y Aitchison, 1997; PhiLlips, 2008).

c) Realizar una serie de actividades vocales en la educación secundaria (conducentes a mejorar la capacidad auditiva de los alumnos en relación a la emisión vocal en grupo) a través de repertorios vocales atractivos y adecuados para estas edades (REAMES, 2001; ELORRIAGA, 2008).

d) La definición práctica de una estrategia efectiva para clasificar y vocalizar las voces de los alumnos adolescentes de ambos sexos que están inmersos en la muda de la voz (WHITE y WHITE, 2001; FREER, 2009b).

e) La conformación de metodologías de apoyo con los que tienen más problemas de coordinación vocal y auditiva y el diseño de ejercicios específicos para este tipo de alumnos (WELCH, 1985).

Desgraciadamente, nada de esto se menciona en ninguno de los textos legales de nuestro actual currículo nacional. Es urgente, por lo tanto, comenzar a construir una didáctica de la voz en la educación secundaria, de modo secuenciado, ordenado, lógico y pedagógico, por supuesto. Una metodología fundamentada epistemológicamente del canto coral en la educación secundaria está aún por desarrollarse en nuestro país. Como observa Helen KEMP (1985), el canto es una destreza que se aprende. Y si el canto es algo que se aprende, es porque es posible enseñar a cantar a cualquier edad, independientemente del contexto académico donde este aprendizaje se lleve a cabo.

\section{Referencias}

Aвitbol, J.; Aвiтвоl, P.; AвiтBol, B. (1999): "Sex hormones and the female voice". Journal of Voice, 13(3), 424-446.

Aguilar, M. C. (2007): El taller coral: Técnicas de armonización vocal para coros principiantes. Buenos Aires, María del Carmen Aguilar.

BARLOW, C.; HowARD, D. M. (2002): "Voice source changes of child and adolescent subjects undergoing singing training-a preliminary study”. Logopedics Phoniatrics Vocology, 27, 6673.

BARLOW, C.; HowARD, D. M. (2005): "Electrolaryngographically derived voice source changes of child and adolescent singers". Logopedics Phoniatrics Vocology, 30, 147-157.

BARlOW, C.; HowARD, D. M. (2007, 11-13 de Julio): "Measured characteristics of development in adolescent singers”. Actas de Sound and Music Computing Conference (págs. 101-106). Lefkada, Grecia.

CAIN, T. (2008): “The characteristics of action research in music education”. British Journal of Music Education, 25(3), 283-313.

Calvo, M. T. M.; Román, J. P. (2007): “Alteraciones endocrinometabólicas más frecuentes en la adolescencia”. Revista Española de Pediatría, 63(1), 38-51.

CÁmArA , A. (2004): "La actividad de cantar en la escuela: Una práctica en desuso". Revista de Psicodidáctica, 17, 1-11.

COOKSEY, J. M. (1977a): "Development of a contemporary, eclectic theory of the training and cultivation of the junior high school male changing voice. Part I: existing theories”. The Choral Journal, 18(2), 5-14.

CoOKSEY, J. M. (1977b): "Development of a contemporary, eclectic theory of the training and cultivation of the junior high school male changing voice. Part II: scientific and empirical findings: some tentative solutions”. The Choral Journal, 18(3), 5-16. 
CoOKSEY, J. M. (1977c): "Development of a contemporary, eclectic theory of the training and cultivation of the junior high school male changing voice. Part III: developing an integrated approach to the care and training of the junior high school male changing voice”. The Choral Journal, 18(4), 5-15.

CoOKSEY, J. M. (1978): "Development of a contemporary, eclectic theory of the training and cultivation of the junior high school male changing voice. Part IV: selecting music for the junior high school male changing voice”. The Choral Journal, 18(5), 5-17.

Cooksey, J. M. (1992): Working with the adolescent voice. St. Louis, Concordia.

Cooksey, J. M. (1993): “Do adolescent voices ‘break’ or do they ‘transform’?” Voice, 2, 15-39.

Demorest, S. M.; Clements, A. (2007): "Factors influencing the pitch-matching of junior high boys”. Journal of Research in Music Education, 55(3), 190-203.

ElorRiagA, A. (2008): El sonido se comparte: Canto colectivo y educación musical. Madrid, UAM Ediciones.

ElorriagA, A. (2010a): "El cambio de la voz masculina en la adolescencia". Eufonía. Didáctica de la Música, 49, 94-105

ElorriagA, A. (2010b): "La muda de la voz femenina durante la adolescencia". Música y Educación, 83, 52-56.

ElorriagA, A. (2010c, 24-26 de junio): "La construcción de la identidad de género en la adolescencia a través del canto coral”. En G. RusineK; M. E. RiAÑO; N. OriOL (eds.): Actas del Seminario Internacional de Investigación en Educación Musical 2010 [cd-rom]. Madrid: SEM-EE - Universidad Complutense de Madrid.

Freer, P. K. (2009a): “Boys’ descriptions of their experiences in choral music”. Research Studies in Music Education, 31(2), 1-19.

FREER, P. K. (2009b): “Choral warm-ups for changing adolescent voices”. Music Educators Journal, 95, 57-62.

Fuchs, M.; FrÖElich, M.; Hentschel, B.; Stuermer, I. W.; Kruse, E.; KnAUfT, D. (2001): "Predicting mutational change in the speaking voice of boys"”. Journal of Voice, 21(2), 169178.

Fuchs, M.; MeURET, S.; GeISTER, D.; PfHOL, W.; THIEL, S.; DIETZ, A. (2008): “Empirical criteria for establishing a classification of singing activity in children and adolescents". Journal of Voice, 22(6), 649-657.

García, M. V. (1847): École de Garcia. Traité complet de l'art du chant en duex parties. París, Troupenas.

GARCíA, M. V. (1894): Hints of singing. New York, Ascherberg.

GACKLE, L. (1985): “The young adolescent female voice (ages 11-15): Classification, placement, and development of tone”. The Choral Journal, 25(8), 15-18.

GACKLE, M. L. (1991): “The adolescent female voice: Characteristics of change and stages of development”. The Choral Journal, 31(8), 17-25.

Hargreaves, D. J. (1998): Música y desarrollo psicológico. Barcelona, Graó.

HARRIS, R. L. (1987): “The young female voice and alto”. The Choral Journal, 28(3), 21-22.

KEMP, H. (1985): Understanding and developing the child's singing voice. St. Louis (Missouri), Concordia. 
MADDOX, D. L. (1986): “A study for developing the head voice to improve pitch-singing accuracy of adolescent girls classified as non-singers”. Missouri Journal of Research in Music Education, 5(3), 123-124.

MiNisTERIO DE EDUCACIÓN Y CIENCIA (2006): Ley Orgánica 2/2006, de 3 de mayo, de Educación. Madrid, Boletín Oficial del Estado.

Meurer, E. M.; Garcez, V.; VON Eye CorletA, H.; CAPP, E. (2007): “Menstrual cycle influences on voice and speech in adolescent females”. Journal of Voice, 23(1), 109-113.

MonKs, S. (2003): “Adolescent singers and perceptions of vocal identity”. British Journal of Music Education, 20(3), 243-256.

MuÑOz, E. (2007): La comprensión musical de las estéticas del s. XX en el niño. Madrid, UAM Ediciones.

PhILliPS, K. H. (1996): Teaching kids to sing. New York, Schirmer.

Phillips, K. H.; Aitchison, R. E. (1997): "Effects of psychomotor instruction on elementary general music students’ singing performance”. Journal of Research in Music Education, 45(2), 185-196.

PHILliPS, M. J. (2008): “Where the wild things are - Teaching middle school boys' choirs”. ChorTeach, 1(1), 4-6. Consultado en http://acda.org/files/ChorTeach_vol.1_Philips,M.pdf.

PRICE, H. E. (1994): "Effects of male timbre, falsetto, and sine-wave models on interval matching by inaccurate singers”. Journal of Research in Music Education, 42(4), 269-284.

Puyuelo Sanclemente, M. (1997): Casos clínicos en logopedia. Barcelona, Elsevier Masson.

REAMES, R. (2001): "High school choral directors' description of appropriate literature for beginning high school choirs”. Journal of Research in Music Education, 49(2), 122-135.

GUIBERT, I. (1992): Optativas: Canto coral. (Materiales para la reforma). Madrid, MEC.

RoBerts, B. (1994): “Music teachers as researchers”. International Journal of Music Education, 23, 24-33.

RusineK, G. (2008): "Disaffected learners and school musical culture: An opportunity for inclusion”. Research Studies in Music Education, 30(1), 9-23.

WeLCH, G. F. (1979): “Vocal range and poor pitch singing” Psychology of Music, 7(2), 13-31.

WELCH, G. F. (1985): “A schema theory of how children learn to sing in tune”. Psychology of Music, 13(1), 3-18.

White, C. D.; White, D. K. (2001): “Commonsense training for changing male voices”. Music Educators Journal, 87(6), 39-43.

WHITEHEAD, J. (1989): “Creating a living educational theory from questions of the kind: How do I improve my practice?” Cambridge Journal of Education, 19, 137-153.

Willis, E. C.; Kenny, D. T. (2007a, 15-19 de agosto): "Phonational gaps in the developing male adolescent voice”. En R. P. K. MAimets-Volt, M. MARIN \& J. Ross (ed.): Conference on Interdisciplinary Musicology (págs. 1-5). Tallinn, Estonia, Australian Centre for Applied Research in Music Performance.

WiLlis, E. C.; KenNy, D. T. (2007b, 5-7 de diciembre): "Variability in speaking fundamental frequency in the adolescent voice”. Comunicación presentada en Conference on Music Communication Science. University of Sidney, Australian Centre for Applied Research in Music Performance. 
WiLlis, E. C.; KENNY, D. T. (2008): "Effect of voice change on singing pitch accuracy in young male singers”. Journal of Interdisciplinary Music Studies, 2(1), 111-119.

Manuscrito recibido el 12/1/2010 y evaluado anónimamente. Aceptado para su publicación el 4/10/2010 